\title{
Optimization of electron bunch quality using a chirped laser pulse in laser wakefield acceleration
}

\author{
Arohi Jain $\odot$ and Devki Nandan Gupta๑* \\ Department of Physics and Astrophysics, University of Delhi, Delhi-110007, India
}

(Received 5 August 2021; accepted 5 November 2021; published 29 November 2021)

\begin{abstract}
In this work, we perform particle-in-cell simulations to investigate the electron bunch quality using a chirped laser pulse in a laser wakefield acceleration mechanism. Particular attention is devoted to the electron beam quality improvement using a chirped laser pulse through group delay dispersion. By adjusting the value of group delay dispersion, the electron beam quality was controlled and improved in the range: charge (31-325 pC), energy (240-485 MeV), energy spread (2.1\%-12.5\%), and bunch duration (3.3-17 fs). The electron bunch charge highly depends on the chirping of the laser pulse. We anticipate that the ultrashort sub-GeV electron bunch generated in the laser wakefield acceleration mechanism using chirped laser pulses may be crucial in developing free-electron laser-based compact radiation sources.
\end{abstract}

DOI: $10.1103 /$ PhysRevAccelBeams.24.111302

\section{INTRODUCTION}

Over the past several decades, laser wakefield acceleration (LWFA) [1] has attracted extensive attention and has been the subject of intense investigation due to its potential applications in soft $\mathrm{x}$-ray/gamma-ray source development $[2,3]$. An intense laser pulse can derive electrostatic wakefields in plasmas, sustaining accelerating gradients exceeded by the orders of magnitude those of conventional particle accelerators. This LWFA technique offers a path towards highly compact and cost-effective accelerators. The laser wakefield accelerations of electron beams have reached to $\mathrm{GeV}$ energy level in only centimeter scales [4]. In fact, femtosecond-long electron bunches with kiloampere current [5] and micron-level emittance can be generated $[6,7]$. The LWFAs are thus becoming a promising option for a wide range of applications such as free-electron lasers (FELs) [8-10] or future linear colliders [11], which would strongly benefit from reduced size and cost. Among other applications, there is also special interest in the production of subfemtosecond bunches to generate short $\mathrm{x}$-ray pulses for ultrafast science [12]. Thus, exploring mechanisms that can control the electron beam properties has been one of the most significant advances still needed for these applications to become practical.

In LWFA, the interaction of intense ultrashort laser pulses with plasma forms an electron density bubble

\footnotetext{
*dngupta@physics.du.ac.in
}

Published by the American Physical Society under the terms of the Creative Commons Attribution 4.0 International license. Further distribution of this work must maintain attribution to the author(s) and the published article's title, journal citation, and DOI. trailing the relativistically intense driver pulse. The laser ponderomotive force creates complete electron cavitation, forming a bubble behind the driver and stripped ions remain immobile due to their high inertia. This ion cavity is commonly referred to as bubble or blowout $[13,14]$. Most of the experiments are performed in the blowout regime to generate a good quality electron bunch in LWFA. To control the electron beam parameters in this regime, various schemes have been investigated and controlled injection has been successfully demonstrated [15-17].

The temporal profile of the laser pulse is modified by changing the spectral phase terms of the laser [18]. The spectral phase term $(\phi(\omega))$ can be expanded in terms of the Taylor series around the central frequency of the laser pulse. The second-order term, $\phi^{(2)}=\partial^{2} \phi / \partial \omega^{2}$, is the spectral phase term describing linear chirp, also defined as the group delay dispersion. Experimentally, a fully optical and programmable method can be used to adjust the specific component of the spectral phase [19-21]. In this study, the energy of the laser pulse remains the same while introducing the linear chirp to the laser profile. To maintain the laser pulse energy, the normalized laser intensity is reduced and pulse duration is stretched by a stretching factor $(s)$ for the specific value of group delay dispersion $\left(\phi^{(2)}\right)$. The laser profile strongly affects the injection process of the electron bunches in the plasma [22-25]. The change in the wake phase velocity via negative density gradient which triggers self-injection is influenced by the laser amplitude. The stronger accelerating field from higher laser amplitude gives higher momenta to the electrons, thus more electrons can overcome the injection threshold for higher $a_{0}$, where $a_{0}=e|\mathbf{E}| /\left(m_{e} \omega c\right)$ is the normalized laser strength parameter of laser with electric field $\mathbf{E}$ and frequency $\omega$. Due to this effect, the 
number of injected electrons increases with $a_{0}$. However, with the growth of the injected electron bunch charge, the self-fields of the injected bunch become more important, which change the structure of the accelerating field, and LWFA begins to operate in the beam loading regime [26-28]. With uncontrolled beam loading, the modification of the accelerating electric field significantly impacts the injection and acceleration process, and this results in reduction of the electron beam quality. Therefore, to produce high-quality electron beams appropriate control over injection of electrons is of great importance. Electron beam quality in LWFA is very sensitive to the laser-plasma parameters; therefore, the stabilization of the electron beam is still a concern of interest. The laser profile is one of the parameters to optimize the electron bunch parameters.

Previous investigations have discussed the important effects of chirp on the generation of wakefield and electron acceleration [29-32]. The effect of chirp on the process of electron injection via down-ramp injection still needs to be explored. In the present study, plasma density transition is used for controlled localized injection and the laser intensity is below the injection threshold to avoid the uncontrolled self-injection. Under this controlled injection scheme, varying group delay dispersion $\left(\phi^{(2)}\right)$ or linear chirp can produce better quality electron bunches preferred for FEL applications. However, reducing the plasma density may limit beam loading effects while keeping laser amplitude maximum. As far as experimental setup is concerned, tuning density bump parameters are experimentally more challenging than varying $\phi^{(2)}$. In this article, group delay dispersion $\phi^{(2)}$ is exploited as an additional mechanism to control the bunch properties of self-injected electrons.

In this article, we particularly give attention to the improvement of electron beam quality using a chirped laser pulse. We explore the control over electron beam parameters by tuning the second-order dispersion in the spectral phase with respect to the optical frequency of the laser pulse. We present the results of systematic particle-incell studies, which give deeper insight into the scaling of electron bunch properties with the intensity and profile of a laser pulse. The plasma density gradient scheme has been adopted to control the phase velocity of the bubble for electron injection [7,33,34]. We have fixed the profile of plasma density used in these simulations to study the effect of laser profile on the properties of injected electron bunches, independently. Specifically, this study suggests that the effect of group delay dispersion $\phi^{(2)}$ of the chirped laser pulse is an important controlling parameter in the plasma density gradient scheme. As a result, a highly tunable quasi-monoenergetic electron beam with narrow energy spread, hundreds of $\mathrm{MeV}$ central energy, and tens of pC charge can be produced. Scaled simulations suggest that the electron beam quality can be improved significantly by selecting the proper initial positive chirp parameter of the laser pulse. We use a quasi-three-dimensional particle-in-cell (3D-PIC) simulation code to explore this proposal. The corresponding simulation results are given in Sec. II. Lastly, a conclusion with a summary is discussed in Sec. III.

\section{SIMULATION RESULTS}

To investigate the influence of laser chirp on electron injection and bunch properties in the bubble regime of LWFA, particle-in-cell (PIC) simulations are performed. Quasi-3D FBPIC code is used for simulations carried over a wide range of laser and plasma parameters [35]. A linearly polarized laser pulse with wavelength $\lambda=0.8 \mu \mathrm{m}$, propagating along the $z$ axis is assigned to the simulation box. The size of the simulation box is $60 \times 60 \mu \mathrm{m}$ with the resolution $\Delta z=0.05 \mu \mathrm{m}$ in the longitudinal direction and $\Delta r=0.4 \mu \mathrm{m}$ in the radial direction. A moving window is employed with a simulation box that moves along the $z$ axis at the speed of light $c$. The two azimuthal modes are used in the simulations with $(2,2,4)$ particles per cell in $(z, r, \theta)$. It should be noted that similar results are obtained for a greater number of macroparticles and higher modes. The laser intensity is $I_{0}=4.3 \times 10^{19} \mathrm{~W} / \mathrm{cm}^{2}$ with spot radius $w_{0}=11 \mu \mathrm{m}$. The laser energy is $2.6 \mathrm{~J}$ and remains constant with varying spectral phase term, $\phi^{(2)}$. The plasma density is set to $2.2 \times 10^{18} \mathrm{~cm}^{-3}$ with $20 \mu \mathrm{m}$ long linear ramp before the arrival of the laser pulse. The length of the plasma medium is $4 \mathrm{~mm}$.

Modulation in the plasma density profile leads to a controlled injection of the electrons in the bubble regime. The plasma density profile proposed in Ref. [7] is used in the simulations which causes injection of ultrashort electron bunches. The plasma density profile is given as

$\frac{n}{n_{e}}= \begin{cases}H(z) z / L, & z \leq L \\ 1+H\left(L_{b}-\left|z-z_{i}\right|\right) k \cos ^{2}\left(\pi \frac{z-z_{i}}{2 L_{b}}\right), & z \geq L,\end{cases}$

where $n_{e}$ is the plateau density, $H(z)$ is the Heaviside function, $\mathrm{L}$ is the length of the initial up-ramp, $z_{i}$ is the position of a density peak of the bump introduced with the half-width $L_{b}$, and $k$ is the amplitude of the density modulation which is the ratio of peak density to the plateau density $n_{e}$. This simulated density profile can be achieved in an experiment using supersonic gas nozzles [36]. Over a positive density gradient, bubble length decreases, the phase velocity at the back of the bubble $v_{p}$ increases which suppresses the electron injection completely. Contrarily, the downward density region of the bump lengthens the bubble, reducing the phase velocity at the back of the bubble and hence injection occurs. In the simulations, density modulation parameters $k=0.3$, $L_{b}=50 \mu \mathrm{m}$ and $z_{i}=350 \mu \mathrm{m}$ remain fixed. For analyzing the effect of the chirped laser pulse, the plasma density profile is held identical for all the simulation runs. 
The temporal profile of the laser pulse can be manipulated by changing each spectral phase term. The spectral phase $\phi(\omega)$ is the phase of each frequency in the waveform. It can be expressed as the expansion of the Taylor series around the central frequency of the laser pulse. The group delay dispersion $\phi^{(2)}$ or the second-order dispersion is the second derivative change in the spectral phase with respect to the optical frequency $\omega$, which describes the linear chirp of the laser pulse expressed as $\phi^{(2)}=\partial^{2} \phi / \partial \omega^{2}$. In the case of the positively chirped laser pulse, the frequency composition linearly increases with time and vice versa for the negatively chirped laser pulse. To maintain the equivalent laser energy while introducing the chirp, the FBPIC code automatically reduces laser amplitude and stretches pulse duration. A stretch factor is defined for conserving laser energy which is expressed as $s=1+\left(2 \phi^{(2)} / \tau_{0}^{2}\right)^{2}$, where $\tau_{0}$ is the pulse duration of laser pulse without any chirp. The normalized laser intensity is reduced by $s$ and pulse
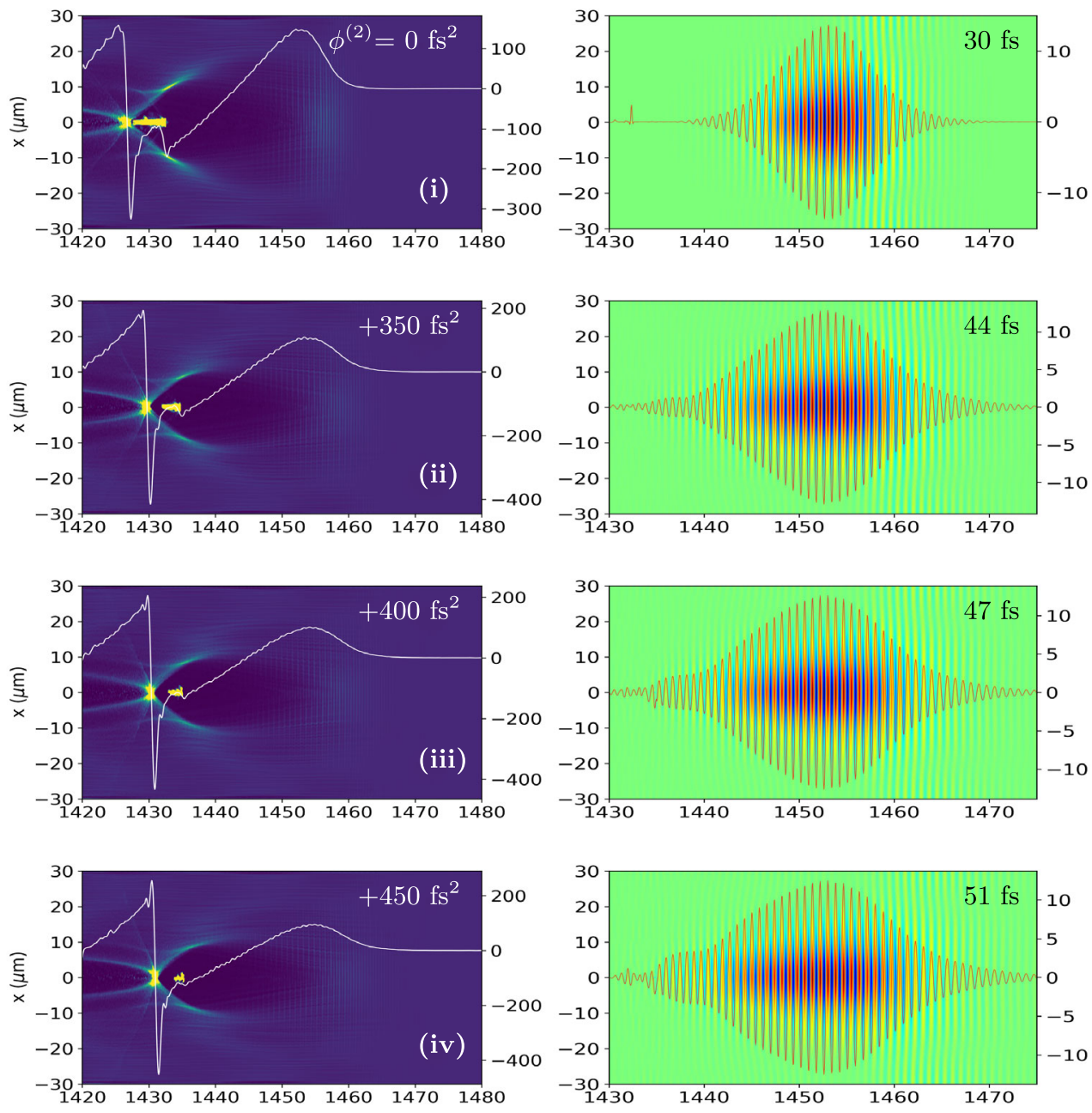

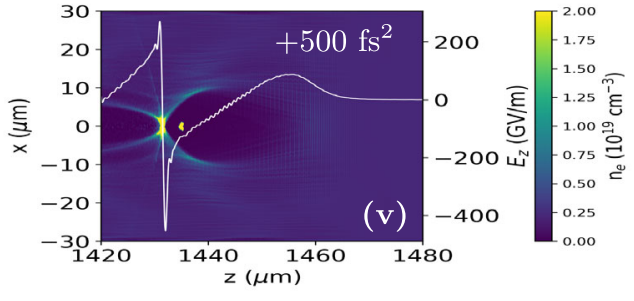

(a)

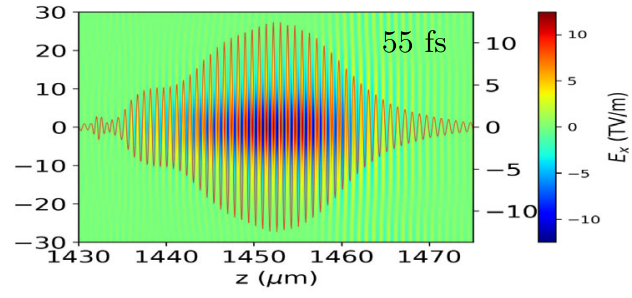

(b)

FIG. 1. Particle-in-cell simulations illustrating controlled electron injection using plasma density modulation. (a) Electron density distribution at different group delay dispersion $\left(\phi^{(2)}\right.$, noted in the graph) of laser pulses. (b) The laser electric field distribution corresponding to $\phi^{(2)}$ with the red line describing the on-axis laser field. The FWHM pulse duration of the laser pulse is indicated in the graph. 
duration is stretched by $s$ for the specific value of group delay dispersion $\phi^{(2)}$. To illustrate the effect of chirped laser pulses on the quality of electron beam, typical values of $\phi^{(2)}$ for positively chirped laser pulses are chosen i.e., $\phi^{(2)}=[0,+150,+250,+350,+380,+400,+450,+500] \mathrm{fs}^{2}$ and the corresponding laser intensity parameters $\left(a_{0}\right)$ and FWHM pulse durations $\left(\tau_{\mathrm{FWHM}}\right)$ are $a_{0}=[4.50,4.30,4.00$, $3.70,3.60,3.56,3.44,3.32]$ and $\tau_{\mathrm{FWHM}}=[30,33,38,44$, $46,47,51,55] \mathrm{fs}$, respectively. The electron density distribution for different group delay dispersion $\phi^{(2)}$ of the laser pulse around laser propagation distance $z=1.5 \mathrm{~mm}$ are shown in Fig. 1(a). For an unchirped laser pulse, we observe an overloaded electron bunch that significantly perturbs the longitudinal electric field. Laser pulse energy remains unchanged for linearly chirped laser pulses. However, introducing group delay dispersion $\phi^{(2)}$ reduces the amplitude of the laser and stretches the pulse duration which decreases the ponderomotive force on the electrons. Varying the chirp of the laser pulse giving us a control over the electron bunch properties. The laser electric field
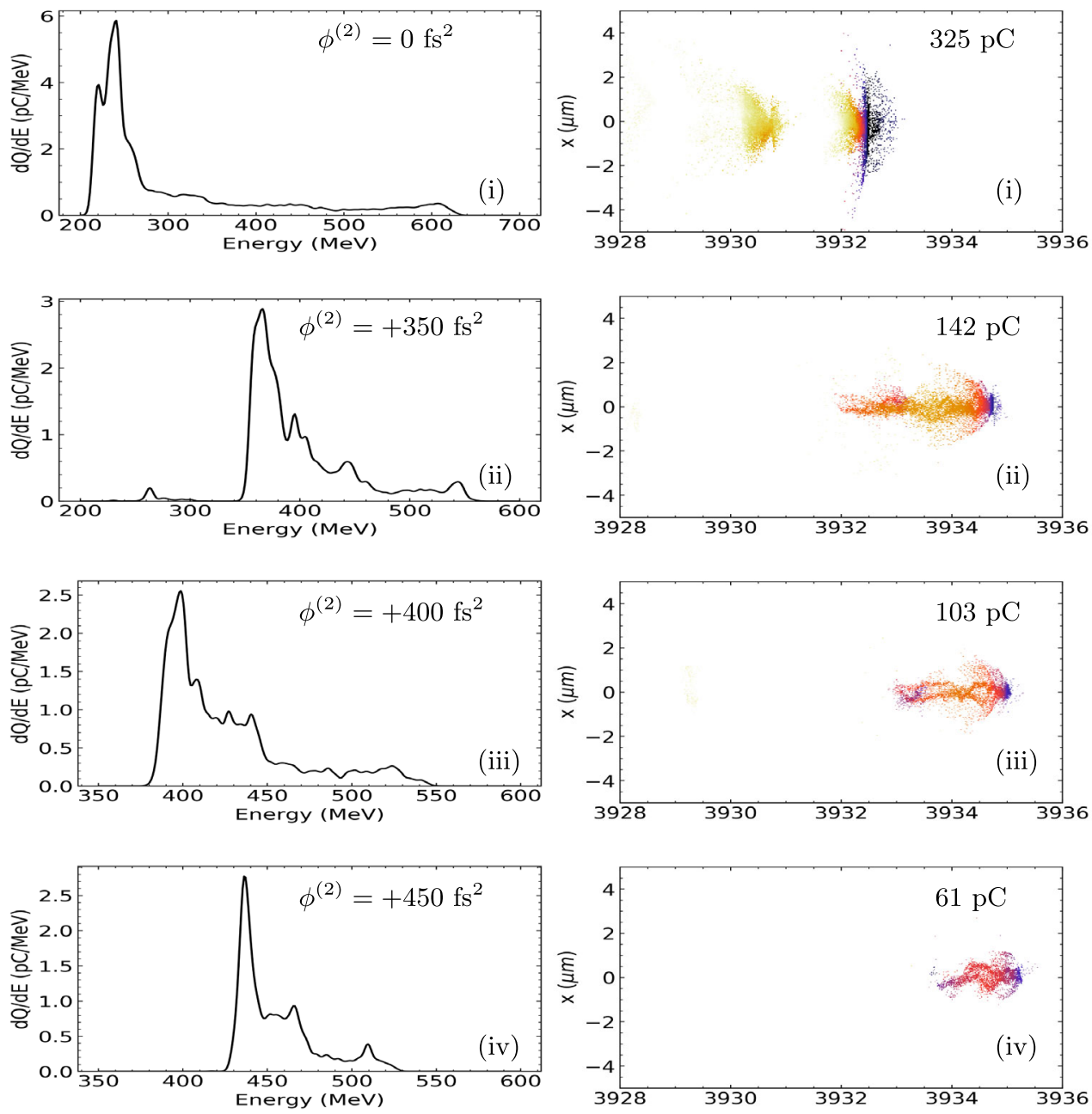

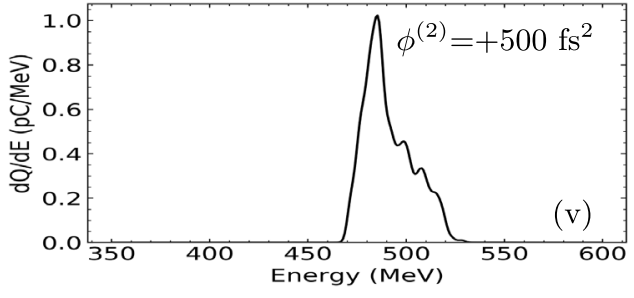

(a)

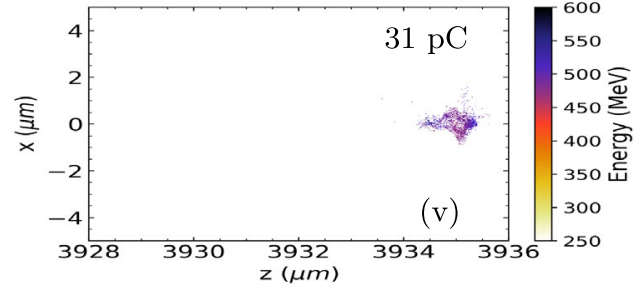

(b)

FIG. 2. (a) Energy spectra for injected electron obtained with different values of the group delay dispersion $\left(\phi^{(2)}\right)$ of the laser pulse. (b) The distribution of electron bunch is represented corresponding to each $\phi^{(2)}$, where the color scheme represents the energy gain by the injected electrons. 
distributions corresponding to each value of $\phi^{(2)}$ are shown in Fig. 1(b). It indicates the lengthening of linearly chirped laser pulses.

The change of electron spectrum and electron energy distribution depending on $\phi^{(2)}$ of the driving laser pulse in a fixed length of plasma are shown in Figs. 2(a) and 2(b), respectively. The electron spectrum produced with an unchirped $\left(\phi^{(2)}=0\right)$ laser pulse is broadband and peaked around $240 \mathrm{MeV}$ as shown in Fig. 2(a). The injected charge strongly decreases with $\phi^{(2)}$ of the driving laser pulse as indicated in Fig. 2(b). However, the obtained energy distributions change from broad spectra to peaked distributions, indicating a strong influence of laser profile on the dynamics of trapping of the electrons and acceleration process. A significant amount of electrons are injected in a chirp-free case which creates a broad energy distribution due to beam loading effects. The electron spectrum shown in Fig. 2 is observed around the same laser propagation distance $z=3.9 \mathrm{~mm}$. The trapping of the electrons is caused by plasma density modulation at a specific position, thus the electrons are injected at the same time and accelerated for almost the same amount of time for different cases. The reduction in pulse intensity and lengthening of laser pulse duration with the increase in the linear chirp of the driving laser pulse reduces the injected charge. The peak energy increases with $\phi^{(2)}$ due to trapped charge reduction, which reduces beam loading effects. It is worth noting that energy spectra with high charge show a strong nonlinear energy chirp and a positively skewed spectrum. In turn of low charge cases (e.g., $\phi^{(2)}=400,450$ and $500 \mathrm{fs}^{2}$ ), the energy spectrum has a quasilinear chirp and is comparatively more symmetric.

The linear chirp parameter $\phi^{(2)}$ is efficient for controlling the phase-space rotation which is essential for the control over the energy spread of the electron bunch. The electron bunch properties for each simulation are shown in Fig. 3 after a propagation distance of $3.6 \mathrm{~mm}$ with respect to the beginning of the plasma density modulation. The average slice energy of the bunch is plotted as a function of $\mathrm{z}$ for four different values of $\phi^{(2)}$ with the splice length of $40 \mathrm{~nm}$.

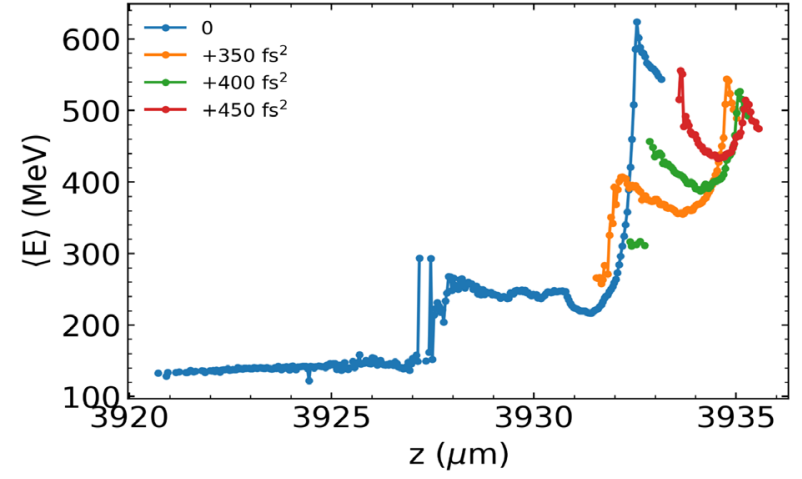

(a)

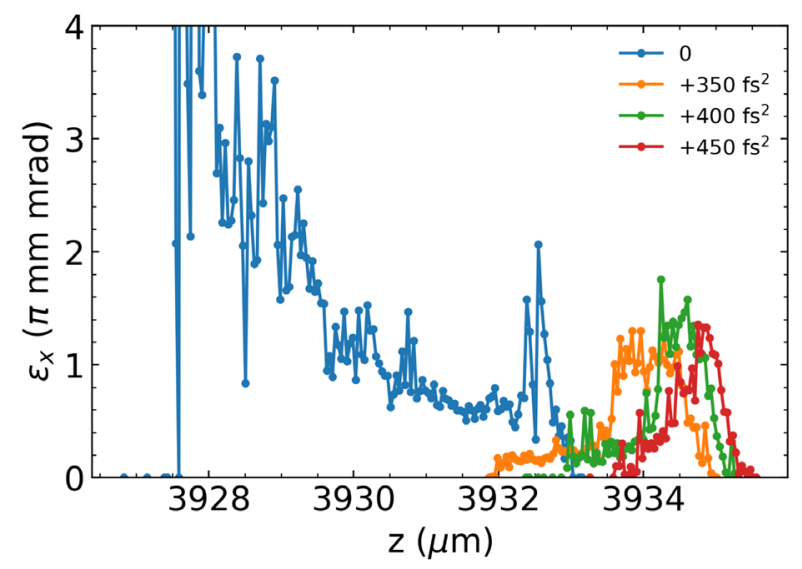

(c)

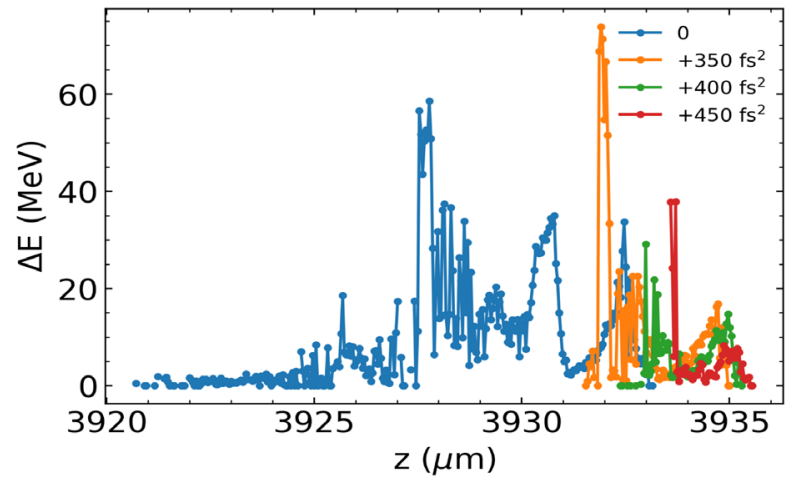

(b)

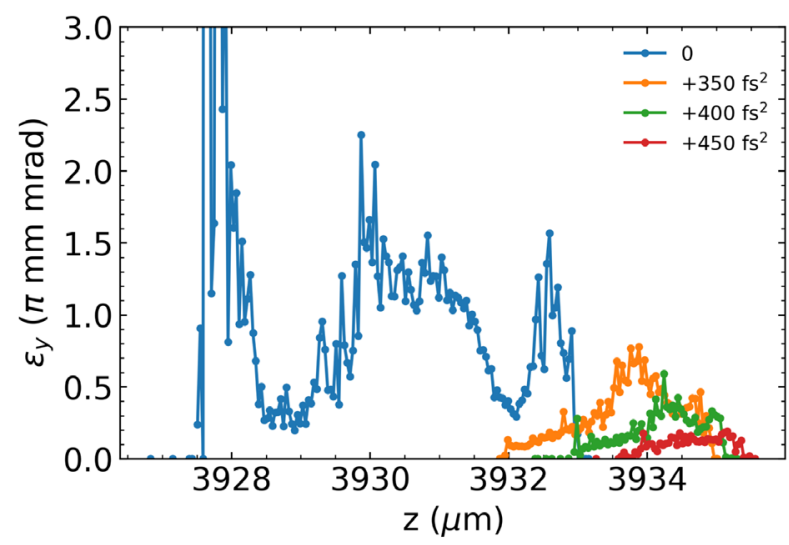

(d)

FIG. 3. The improved bunch quality of accelerated electrons is obtained for a linearly chirped laser pulse. Slice distribution of (a) average electron energy $\langle E\rangle$, (b) energy spread of electrons $\Delta E$, and (c) and (d) normalized rms emittance $\epsilon_{x}$ (in the $x$ direction) and $\epsilon_{y}$ (in the $y$ direction) of the injected electron bunch for different values of group delay dispersion $\left(\phi^{(2)}\right)$ with slice length of $40 \mathrm{~nm}$ around $z=3.9 \mathrm{~mm}$. 
The beam-loaded electric field reduces the amplitude of the accelerating field. As a result, lower energy electrons situated at the tail of the electron bunch never catch the higher energy electrons situated at the head as shown by the distribution of average energy of electrons for $\phi^{(2)}=0$ and $350 \mathrm{fs}^{2}$ in Fig. 3(a). The large separation between low and high energy electrons for these values of $\phi^{(2)}$ leads to a broader energy spread as shown in Fig. 3(b). The analysis of the PIC simulation reveals that the amount of trapped charge leads to a variation in the electron bunch spectrum due to beam loading. The sensitivity of the laser profile is due to the process of injection caused by plasma density modulation which is closely related to the intensity and pulse duration of the laser pulse at the position of density bump. The area in the phase space where charge can be trapped depends on the radius $R$ which is bubble radius or radius of the ion cavity formed behind the laser pulse, which scales as $R \propto \sqrt{a_{0} / n_{e}}[13,37]$, where $a_{0}$ is the normalized laser amplitude defined as $a_{0}=$ $8.5 \times 10^{-10} \sqrt{I\left[\mathrm{~W} / \mathrm{cm}^{2}\right] \lambda^{2}[\mu \mathrm{m}]}$ with $I$ and $\lambda$ being the intensity and wavelength of the laser pulse, respectively. Hence, the injection process and the involved accelerating fields are directly influenced by the intensity reduction and lengthening of the laser pulse. Figure 3(b) suggests that the slice energy spread significantly reduces with $\phi^{(2)}$. The normalized rms emittance $\epsilon_{i}$ along the $i$ direction is defined as $\epsilon_{i}^{2}=\left\langle x_{i}^{2}\right\rangle\left\langle\left(P_{i}\right)^{2}\right\rangle-\left\langle x_{i} P_{i}\right\rangle^{2}$, where $x_{i}$ and $P_{i}$ are the position and normalized momentum of electrons in the transverse plane $(i=[x, y]$ directions), respectively.

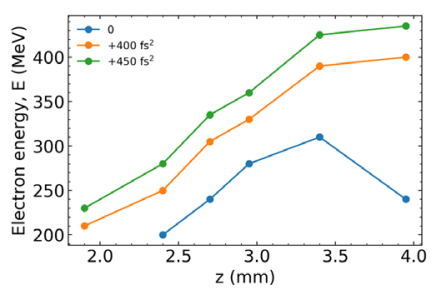

(a)

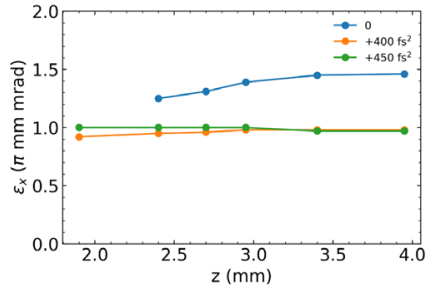

(c) (b)

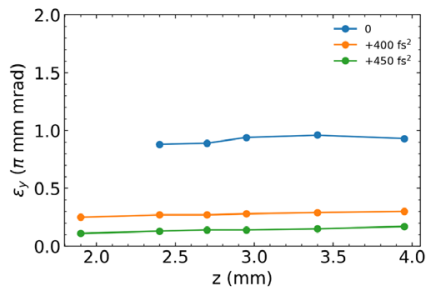

(d)

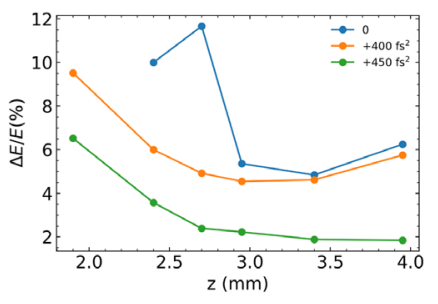

FIG. 4. Evolution of electron bunch properties over the longitudinal traveling distance $z$ : (a) peak energy (E), (b) relative energy spread $(\Delta E / E \%)$, (c) normalized rms values of the emittance in the $x$, and (d) $y$ directions. In each figure, three curves represents different values of group delay dispersion $\left(\phi^{(2)}\right)$ of the laser pulse.
The slice normalized rms emittance is reported in Figs. 3(c) and 3(d). The figures show a clear trend of increasing emittance with the amount of charge (increases with $\phi^{(2)}$ ). The increasing emittance with charge density is a direct evidence of the space charge effects. In general, the inclusion of space charge effects in the description of the transverse dynamics of trapped electrons increases the area of phase space.

In Fig. 4, we show the evolution of the peak energy E, relative energy spread $(\Delta E / E \%)$, and normalized rms emittance $\left(\epsilon_{x}-\epsilon_{y}\right)$ of the injected electron bunches for the selected values of the group delay dispersion $\phi^{(2)}$. We observed from these results that the most interesting electron bunches are obtained when employing the higher values of $\phi^{(2)}$. The acceleration performance is better for $\phi^{(2)}=[400,450] \mathrm{fs}^{2}$ since the injected electron bunches have a lower amount of charge and shorter length. The relative energy spread and correlated normalized emittance are smaller for $\phi^{(2)}=[400,450] \mathrm{fs}^{2}$. Therefore, by varying group delay dispersion values of the laser pulse, one can produce electron bunches with optimized properties.
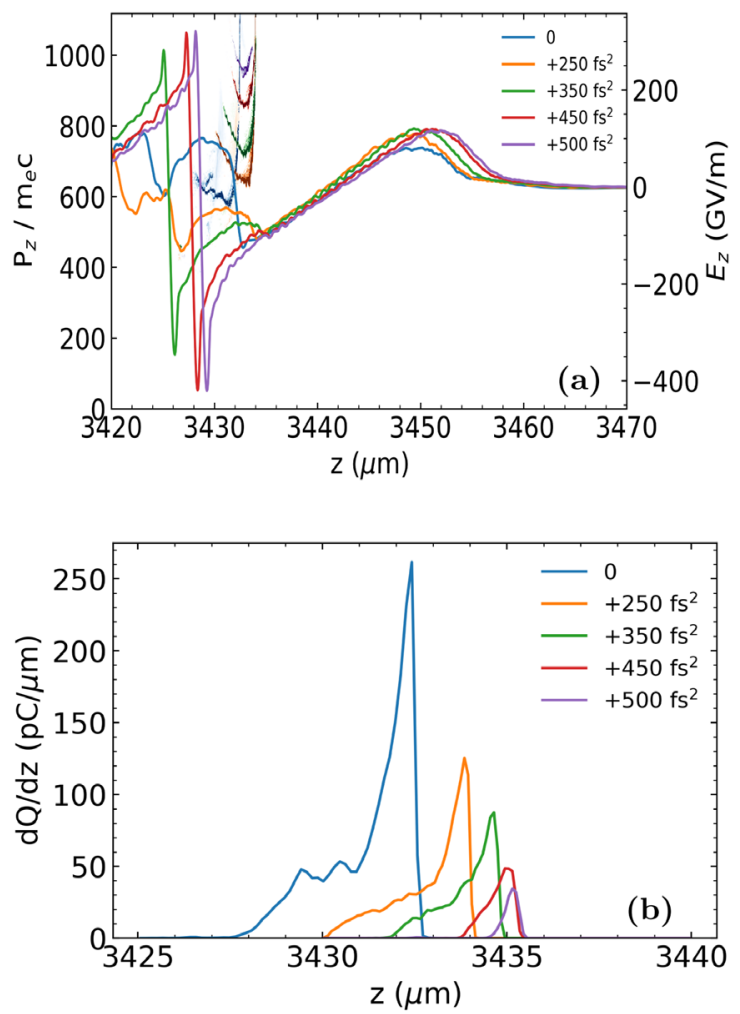

FIG. 5. Results from particle-in-cell simulations for different group delay dispersion $\left(\phi^{(2)}\right)$ values of laser pulse showing: (a) phase space and longitudinal on-axis electric field $E_{z}$, and (b) longitudinal charge distribution of the injected electron bunch around $z=3.5 \mathrm{~mm}$. Electron bunches of different charge are injected by changing the temporal chirp $\left(\phi^{(2)}\right)$ of the laser pulse. Higher charges decrease the accelerating fields of the wakefields due to the effect of beam loading. 


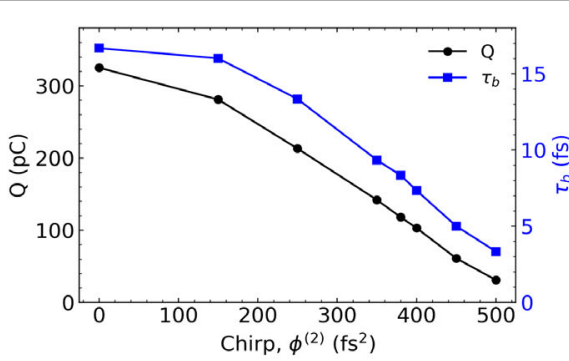

(a)

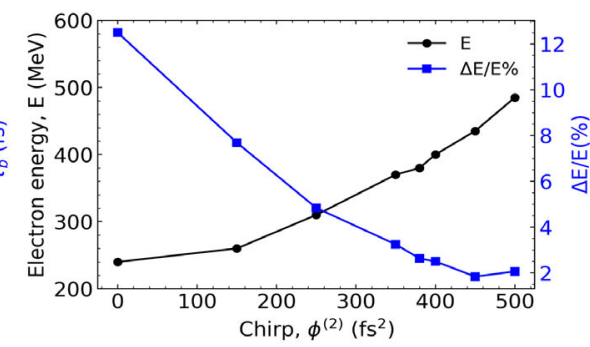

(b)

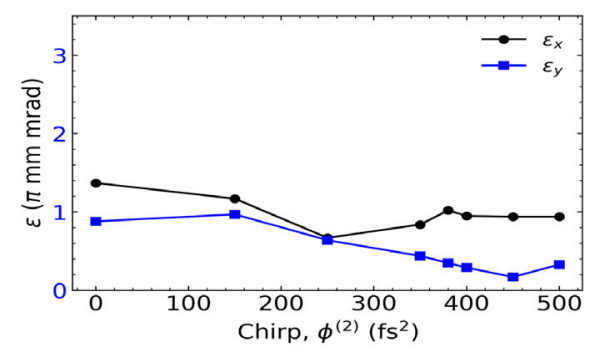

(c)

FIG. 6. Electron bunch properties with respect to the group delay dispersion $\phi^{(2)}$ of a laser pulse. (a) Charge Q (black dots) and electron bunch duration $\tau_{b}$ (blue dots), (b) electron peak energy $\mathrm{E}$ (black dots) and energy spread $\Delta E / E$ (blue dots), and (c) normalized rms emittance $\epsilon_{x}$ (black dots) in the $x$ direction and $\epsilon_{y}$ (blue dots) in the $y$ direction.

Beam loading strongly deforms the accelerating field due to the trapped electrons, influencing the electron bunch at all accelerating stages. Figure 5(a) highlights the beam loading effect on the accelerating field, reporting the deformation in longitudinal electric field when the laser pulse is at position $z \approx 3.5 \mathrm{~mm}$. In Fig. 5(a), the longitudinal electric field is reported for different values of $\phi^{(2)}$ (keeping the laser energy fixed), showing that with decreasing the values of $\phi^{(2)}$, a higher charge is injected (see Fig. 2). Due to the high charge injected, the waveform is much more deformed and the accelerating field experienced by the electron bunch is lower. As expected, the normalized laser amplitude $\left(a_{0}\right)$ decreases with the increase in the values of group delay dispersion $\left(\phi^{(2)}\right)$ : a lower value of $a_{0}$ yields a lower injected charge. The wake phase velocity also depends on the laser amplitude in the nonlinear regimes [7], thus the change in the wake phase velocity due to density modulation triggers the injection (influenced by $a_{0}$ ). Besides this, more electrons can overcome the velocity threshold for injection with the increase in the laser peak field. The electrons gain higher momenta by higher accelerating gradients, which increases the number of injected electrons with higher values of $a_{0}$. In nonlinear regimes, the size of the wakefield bubble structure increases with $a_{0}$ [38]. The wakefield structure reduces with $\phi^{(2)}$ which is confirmed in Fig. 5(a). The length of the injected electron bunch reduces with $\phi^{(2)}$ as shown in Fig. 5(b). The change in electron momenta and bubble size affects the injection volume at the down-ramp, which results in shortening of the bunch duration.

Finally, we report electron beam properties by varying the group delay dispersion $\left(\phi^{(2)}\right)$ of a laser pulse. Electron beam properties include the amount of charge injected, bunch duration, peak energy, energy spread, and emittance in $x-y$ directions (as shown in Fig. 6). A relation between the beam charge and its duration with $\phi^{(2)}$ can be inferred from Fig. 6(a). The bunches with more charge tend to have a longer duration. As stated earlier, the peak amplitude $a_{0}$ of the laser pulse varies with $\phi^{(2)}$. For a given density transition, the bubble velocity depends on $a_{0}$ in nonlinear regimes [39]. Thus the change in the wake phase velocity in the transition triggers the injection (influenced by the laser pulse amplitude). Hence, the electron bunch charge decreases with the group delay dispersion $\phi^{(2)}$. Also, in the nonlinear regimes, the wakefield bubble size expands with $a_{0}$ [38]. For given density transition parameters, higher $a_{0}$ yields longer bunches because the injection starts earlier in the down ramp region of the bump. Due to beam loading,

TABLE I. Tabulation of electron bunch energy (E), energy spread ( $\Delta E / E \%)$, injected bunch charge (Q), bunch duration, transverse normalized rms emittance $\left(\epsilon_{x}-\epsilon_{y}\right)$, and divergence $\left(\theta_{x}-\theta_{y}\right)$ with respect to the group delay dispersion $\left(\phi^{(2)}\right)$ of a laser pulse. The electron beam parameters are reported around $z \approx 3.9 \mathrm{~mm}$ distance traveled by a laser pulse in a plasma.

\begin{tabular}{lcccccc}
\hline \hline$\phi^{(2)}\left(\mathrm{fs}^{2}\right)$ & $\mathrm{Q}(\mathrm{pC})$ & $\mathrm{E}(\mathrm{MeV})$ & $\Delta E / E(\%)$ & $\tau_{b}(\mathrm{fs})$ & $\epsilon_{x}-\epsilon_{y}(\pi \mathrm{mmmrad})$ & $\theta_{x}-\theta_{y}(\mathrm{mrad})$ \\
\hline 0 & 325 & 240 & 12.5 & 16.7 & $1.37-0.88$ & $4.75-3.92$ \\
+150 & 281 & 260 & 7.8 & 16.0 & $1.17-0.97$ & $4.00-3.85$ \\
+250 & 213 & 310 & 4.8 & 13.3 & $0.67-0.64$ & $2.78-2.36$ \\
+350 & 142 & 270 & 3.2 & 9.3 & $0.84-0.44$ & $2.80-2.04$ \\
+380 & 118 & 380 & 2.6 & 8.3 & $1.02-0.35$ & $3.00-1.72$ \\
+400 & 103 & 400 & 2.5 & 7.3 & $0.95-0.29$ & $2.93-1.55$ \\
+450 & 61 & 435 & 1.8 & 5 & $0.94-0.17$ & $2.60-1.14$ \\
+500 & 31 & 485 & 2.1 & 3.3 & $0.94-0.45$ & $3.98-2.47$ \\
\hline \hline
\end{tabular}


higher beam charge (by varying $\phi^{(2)}$ ) reduces the energy gain of the electron bunch as shown in Fig. 6(b). The energy spread decreases with $\phi^{(2)}$, which can also be explained with decrease the electron bunch duration. Because of this, different parts of the bunch would experience different accelerating fields. Thus the energy spread reduces with $\phi^{(2)}$. The normalized rms emittances $\left(\epsilon_{x}-\epsilon_{y}\right)$ in the transverse plane are reported in Fig. 6(c). We observe a stable variation of emittance $\left(\epsilon_{x}-\epsilon_{y}\right)$ with $\phi^{(2)}$. The emittance in the $y$ direction is lower than of the $x$ direction due to the laser polarization.

The previously discussed results demonstrated that the group delay dispersion $\phi^{(2)}$ acts as a crucial parameter for optimizing the electron bunch properties. The effects of group delay dispersion $\phi^{(2)}$ can be summarized in Table I. We found that the optimizing laser parameters are suitable for independent control for essential LWFA mechanisms. This degree of control will be crucial to the applications of laser wakefield accelerators in the development of $\mathrm{x}$-ray sources.

\section{CONCLUSION}

In conclusion, we have investigated the optimization and controlling of the electron bunch parameters in the beam loading regime via group delay dispersion $\phi^{(2)}$ of the laser pulse. In this study, the laser energy remains the same by varying $\phi^{(2)}$, which introduces a linear chirp in the laser pulse. In order to maintain the laser energy, laser intensity parameter $\left(a_{0}\right)$ and FWHM pulse duration $\left(\tau_{\mathrm{FWHM}}\right)$ both increase with $\phi^{(2)}$. The laser amplitude varies with $\phi^{(2)}$, which causes different amount of charge injection and electron bunch duration. Thus the group delay dispersion provides a good control over the bunch parameters and an optimized electron bunch can be generated using a chirped laser pulse. We have obtained a range of values for electron bunch properties listed in Table I. The resulted electron bunches with tunable charge, energy, energy spread, and bunch duration will be crucial to the applications of laser wakefield accelerators. The optimized way of varying laser parameters via $\phi^{(2)}$ provides new physical insights into the injection and acceleration processes through simulations and will result in improved performance of laser-driven electron accelerators.

\section{ACKNOWLEDGMENTS}

The authors would like to thank the Department of Physics and Astrophysics, University of Delhi, India, for providing computational facilities to carry out the simulation work. This is financially supported by the Department of Science and Technology, Government of India (Grant No. INT/RUS/RFBR/394) and the Institution of Eminence (IoE), University of Delhi, under Faculty Research Programme Grant (Ref. No. /IoE/2021/12/FRP).
A. J. would like to thank Council of Scientific and Industrial Research (CSIR), Government of India (File No. 09/045(1510)2017-EMR-I) for providing Senior Research Fellowship (SRF) during the Ph.D. program.

[1] T. Tajima and J. M. Dawson, Laser Electron Accelerator, Phys. Rev. Lett. 43, 267 (1979).

[2] A. Rousse, K. T. Phuoc, R. Shah, A. Pukhov, E. Lefebvre, V. Malka, S. Kiselev, F. Burgy, J. P. Rousseau, D. Umstadter, and D. Hulin, Production of a KeV X-ray Beam from Synchrotron Radiation in Relativistic LaserPlasma Interaction, Phys. Rev. Lett. 93, 135005 (2004).

[3] S. Cipiccia et al., Gamma-rays from harmonically resonant betatron oscillations in a plasma wake, Nat. Phys. 7, 867 (2011).

[4] A. J. Gonsalves et al., Petawatt Laser Guiding and Electron Beam Acceleration to $8 \mathrm{GeV}$ in a Laser-Heated Capillary Discharge Waveguide, Phys. Rev. Lett. 122, 084801 (2019).

[5] M. R. Islam, E. Brunetti, R. P. Shanks, B. Ersfeld, R. C. Issac, S. Cipiccia, M.-P. Anania, G. H. Welsh, S. M. Wiggins, A. Noble, R. A. Cairns, G. Raj, and D. A. Jaroszynski, Near-threshold electron injection in the laser plasma wakefield accelerator leading to femtosecond bunches, New J. Phys. 17, 093033 (2015).

[6] E. Brunetti, R. P. Shanks, G. G. Manahan, M. R. Islam, B. Ersfeld, M. P. Anania, S. Cipiccia, R. C. Issac, G. Raj, G. Vieux, G. H. Welsh, S. M. Wiggins, and D. A. Jaroszynski, Low Emittance, High Brilliance Relativistic Electron Beams from a Laser-Plasma Accelerator, Phys. Rev. Lett. 105, 215007 (2010).

[7] M. P. Tooley, B. Ersfeld, S. R. Yoffe, A. Noble, E. Brunetti, Z. M. Sheng, M. R. Islam, and D. A. Jaroszynski, Towards Attosecond High-Energy Electron Bunches: Controlling Self-Injection in Laser-Wakefield Accelerators through Plasma-Density Modulation, Phys. Rev. Lett. 119, 044801 (2017).

[8] D. A. Jaroszynski and G. Vieux, Coherent radiation sources based on laser plasma accelerators, AIP Conf. Proc. 647, 902 (2002).

[9] H.-P. Schlenvoigt, K. Haupt, A. Debus, F. Budde, O. Jäckel, S. Pfotenhauer, H. Schwoerer, E. Rohwer, J. G. Gallacher, E. Brunetti, R. P. Shanks, S. M. Wiggins, and D. A. Jaroszynski, A compact synchrotron radiation source driven by a laser-plasma wakefield accelerator, Nat. Phys. 4, 130 (2008).

[10] A. R. Maier, A. Meseck, S. Reiche, C. B. Schroeder, T. Seggebrock, and F. Grüner, Demonstration Scheme for a Laser-Plasma-Driven Free-Electron Laser, Phys. Rev. X 2, 031019 (2012).

[11] C. B. Schroeder, E. Esarey, C. G. R. Geddes, C. Benedetti, and W. P. Leemans, Physics considerations for laserplasma linear colliders, Phys. Rev. ST Accel. Beams 13, 101301 (2010).

[12] S. Corde, K. Ta Phuoc, G. Lambert, R. Fitour, V. Malka, A. Rousse, A. Beck, and E. Lefebvre, Femtosecond x rays from laser-plasma accelerators, Rev. Mod. Phys. 85, 1 (2013). 
[13] A. Pukhov and J. Meyer-Ter-Vehn, Laser wakefield acceleration: The highly nonlinear broken-wave regime, Appl. Phys. B 74, 355 (2002).

[14] W. Lu, M. Tzoufras, C. Joshi, F. S. Tsung, W. B. Mori, J. Vieira, R. A. Fonseca, and L. O. Silva, Generating multi$\mathrm{GeV}$ electron bunches using single stage laser wakefield acceleration in a $3 \mathrm{~d}$ nonlinear regime, Phys. Rev. ST Accel. Beams 10, 061301 (2007).

[15] J. Faure, C. Rechatin, A. Norlin, A. Lifschitz, Y. Glinec, and V. Malka, Controlled injection and acceleration of electrons in plasma wakefields by colliding laser pulses, Nature (London) 444, 737 (2006).

[16] P. Tomassini, D. Terzani, F. Baffigi, F. Brandi, L. Fulgentini, P. Koester, L. Labate, D. Palla, and L. A. Gizzi, High-quality $5 \mathrm{GeV}$ electron bunches with resonant multipulse ionization injection, Plasma Phys. Controlled Fusion 62, 014010 (2019).

[17] A. Jain and D. N. Gupta, Improvement of electron beam quality in laser wakefield acceleration by a circularly polarized laser pulse, Plasma Phys. Controlled Fusion 63, 075007 (2021).

[18] A. Weiner, Ultrafast Optics (John Wiley and Sons, New York, 2009).

[19] P. Tournois, Acousto-optic programmable dispersive filter for adaptive compensation of group delay time dispersion in laser systems, Opt. Commun. 140, 245 (1997).

[20] T. Oksenhendler, S. Coudreau, N. Forget, V. Crozatier, S. Grabielle, R. Herzog, O. Gobert, and D. Kaplan, Selfreferenced spectral interferometry, Appl. Phys. B 99, 7 (2010).

[21] C. Liu, J. Zhang, S. Chen, G. Golovin, S. Banerjee, B. Zhao, N. Powers, I. Ghebregziabher, and D. Umstadter, Adaptive-feedback spectral-phase control for interactions with transform-limited ultrashort high-power laser pulses, Opt. Lett. 39, 80 (2014).

[22] H. T. Kim, V. B. Pathak, K. Hong Pae, A. Lifschitz, F. Sylla, J. H. Shin, C. Hojbota, S. K. Lee, J. H. Sung, H. W. Lee, E. Guillaume, C. Thaury, K. Nakajima, J. Vieira, L. O. Silva, V. Malka, and C. H. Nam, Stable multi-GeV electron accelerator driven by waveform-controlled PW laser pulses, Sci. Rep. 7, 1 (2017).

[23] D. E. Mittelberger, M. Thévenet, K. Nakamura, A. J. Gonsalves, C. Benedetti, J. Daniels, S. Steinke, R. Lehe, J.-L. Vay, C. B. Schroeder, E. Esarey, and W. P. Leemans, Laser and electron deflection from transverse asymmetries in laser-plasma accelerators, Phys. Rev. E 100, 063208 (2019).

[24] K. Gopal, D. N. Gupta, and H. Suk, Pulse-length effect on laser wakefield acceleration of electrons by skewed laser pulses, IEEE Trans. Plasma Sci. 49, 1152 (2021).

[25] K. Gopal, D. N. Gupta, A. Jain, M. S. Hur, and H. Suk, Investigation of electron beam parameters in laser wakefield acceleration using skewed laser pulse and external magnetic field, Curr. Appl. Phys. 25, 82 (2021).
[26] T. C. Katsouleas, S. Wilks, P. Chen, J. Dawson, and J. Su, Beam loading in plasma accelerators, Part. Accel. 22, 81 (1987).

[27] M. Tzoufras, W. Lu, F. S. Tsung, C. Huang, W. B. Mori, T. Katsouleas, J. Vieira, R. A. Fonseca, and L. O. Silva, Beam Loading in the Nonlinear Regime of Plasma-Based Acceleration, Phys. Rev. Lett. 101, 145002 (2008).

[28] C. Rechatin, X. Davoine, A. Lifschitz, A. B. Ismail, J. Lim, E. Lefebvre, J. Faure, and V. Malka, Observation of Beam Loading in a Laser-Plasma Accelerator, Phys. Rev. Lett. 103, 194804 (2009).

[29] X. Zhang, B. Shen, L. Ji, W. Wang, J. Xu, Y. Yu, L. Yi, X. Wang, N. A. M. Hafz, and V. Kulagin, Effect of pulse profile and chirp on a laser wakefield generation, Phys. Plasmas 19, 053103 (2012).

[30] V. B. Pathak, J. Vieira, R. A. Fonseca, and L. O. Silva, Effect of the frequency chirp on laser wakefield acceleration, New J. Phys. 14, 023057 (2012).

[31] F. Sohbatzadeh and H. Akou, Group velocity dispersion and relativistic effects on the wakefield induced by chirped laser pulse in parabolic plasma channel, Phys. Plasmas 20, 043101 (2013).

[32] Y. Cui, G.-B. Zhang, Y.-Y. Ma, D.-B. Zou, X.-H. Yang, M. Chen, J.-X. Liu, Z.-Y. Ge, L.-C. Tian, L.-F. Gan, and F.-Q. Shao, Beam quality improvement of ionization injected electrons by using chirped pulse in wakefield acceleration, Plasma Phys. Controlled Fusion 61, 085023 (2019).

[33] C. Zhang, C.-K. Huang, K. A. Marsh, X. L. Xu, F. Li, M. Hogan, V. Yakimenko, S. Corde, W. B. Mori, and C. Joshi, Effect of fluctuations in the down ramp plasma source profile on the emittance and current profile of the selfinjected beam in a plasma wakefield accelerator, Phys. Rev. Accel. Beams 22, 111301 (2019).

[34] L. T. Ke, K. Feng, W. T. Wang, Z. Y. Qin, C. H. Yu, Y. Wu, Y. Chen, R. Qi, Z. J. Zhang, Y. Xu, X. J. Yang, Y. X. Leng, J. S. Liu, R. X. Li, and Z. Z. Xu, Near-GeV Electron Beams at a Few Per-Mille Level from a Laser Wakefield Accelerator via Density-Tailored Plasma, Phys. Rev. Lett. 126, 214801 (2021).

[35] R. Lehe, M. Kirchen, I. A. Andriyash, B. B. Godfrey, and J. L. Vay, Comput. Phys. Commun. 203, 66 (2016).

[36] K. Schmid and L. Veisz, Supersonic gas jets for laserplasma experiments, Rev. Sci. Instrum. 83, 053304 (2012).

[37] E. Esarey, C. B. Schroeder, and W. P. Leemans, Physics of laser-driven plasma-based electron accelerators, Rev. Mod. Phys. 81, 1229 (2009).

[38] C. Benedetti, C. B. Schroeder, E. Esarey, F. Rossi, and W. P. Leemans, Numerical investigation of electron selfinjection in the nonlinear bubble regime, Phys. Plasmas 20, 103108 (2013).

[39] G. Golovin, S. Chen, N. Powers, C. Liu, S. Banerjee, J. Zhang, M. Zeng, Z. Sheng, and D. Umstadter, Tunable monoenergetic electron beams from independently controllable laser-wakefield acceleration and injection, Phys. Rev. ST Accel. Beams 18, 011301 (2015). 